\title{
Catheter-Directed Thrombolysis Along with Mechanical Thromboaspiration versus Anticoagulation Alone in the Management of Lower Limb Deep Venous Thrombosis- A Comparative Study
}

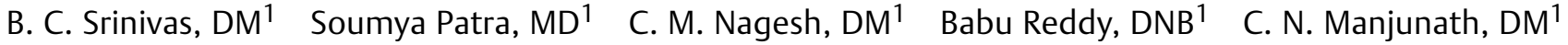 \\ ${ }^{1}$ Department of Cardiology, Sri Jayadeva Institute of Cardiovascular \\ Sciences and Research, Bangalore, Karnataka, India \\ Int J Angiol 2014;23:247-254.

\begin{abstract}
Address for correspondence Soumya Patra, MD, Department of Cardiology, Sri Jayadeva Institute of Cardiovascular Sciences and Research, Bannerghatta Road, Bangalore, Karnataka 560069, India (e-mail: dr_soumyapatra@rediffmail.com).
\end{abstract}

\begin{abstract}
\section{Keywords}

- catheter-directed thrombolysis

- deep vein thrombosis

- iliofemoral

- postthrombotic syndrome

- streptokinase

Catheter-directed thrombolysis (CDT) with assisted mechanical thrombolysis is now considered as the standard of medical care for deep vein thrombosis (DVT). The study was conducted to describe the immediate and long-term (6 months) safety and effectiveness of CDT in patient with lower limb DVT compared with the routine anticoagulation alone. All 12 to 85 years old patients with recent (0-8 weeks) DVT were included. In CDT group, thrombus was aspirated mechanically and streptokinase (STK) was given along with unfractionated heparin (UFH). After 6 months, deep venous patency and postthrombotic syndrome (PTS) was assessed by using duplex ultrasound and Villalta scale, respectively. Among 51 patients with completed data, 25 patients were allocated additional CDT given for a mean duration of $108 \pm 32$ hours and 26 patients were allocated standard treatment alone. Grade III (complete) lysis was achieved in $37 \%$ patients and grade II (50-90\%) lysis in $63 \%$ of patients. Patients with partial lysis underwent percutaneous transluminal angioplasty and/or venous stenting. After 6 months, iliofemoral patency was found in $20(80 \%)$ in the CDT group versus 7 $(23 \%)$ in anticoagulation alone group $(p<0.01)$. PTS was seen in $5(20 \%)$ in the CDT group versus $19(77 \%)$ in anticoagulation alone group $(p<0.01)$. We conclude that CDT and conventional manual aspiration thrombectomy are an effective treatment for lower extremity DVT. STK infusion can be safely given up to 6 days. As addition of UFH can cause thrombocytopenia, so daily monitoring of complete blood counts is needed during CDT.
\end{abstract}

Deep vein thrombosis (DVT) is the third most common cardiovascular pathology after coronary artery disease and stroke. ${ }^{1,2}$ Its incidence is expected to rise over the next decade due to the rising elderly population and increased exposure to DVT risk factors such as hospital admission, oral contraceptives, pregnancy, obesity, and long distance travel. ${ }^{3,4}$ Patients with symptomatic lower extremity venous thromboembolism (VTE), particularly in the iliofemoral DVT, are among those who are more likely to develop lifelong adverse clinical consequences as well as being the most frequently hospitalized for treatment. $^{5,6}$ Though the treatment of VTE has advanced significantly in recent years, ${ }^{7}$ still 20 to $80 \%$ of patients develop postthrombotic syndrome (PTS) despite adequate anticoagulation therapy. ${ }^{8}$ In the area of venous thromboembolic disease, catheter-directed thrombolysis (CDT) with or without assisted mechanical thrombolysis is nowadays becoming the standard published online August 19, 2014
Copyright $\odot 2014$ by Thieme Medical Publishers, Inc., 333 Seventh Avenue, New York, NY 10001, USA. Tel: +1(212) 584-4662.
DOI http://dx.doi.org/ 10.1055/s-0034-1382157. ISSN 1061-1711. 
of medical care in the treatment of acute and subacute DVT ${ }^{9}$ as anticoagulation therapy alone is ineffective at removing thrombus from the deep venous system. ${ }^{10}$ Aggressive therapy to remove the venous thrombus/venous obstruction is required to avoid the development of PTS. ${ }^{11}$ Endovascular management using percutaneous mechanical thrombectomy alone or in combination with pharmacological thrombolytic agents has recently received much attention in the literature as a safe and effective means for the treatment of acute DVT. ${ }^{12,13}$ This study was undertaken to compare the efficacy of CDT and pharmacomechanical thrombolysis, which are usually the first line of therapy for early removal of an acute thrombus in the proximal veins of the leg with routine anticoagulation alone.

\section{Subjects and Method}

\section{Primary End Point}

Primary end point of this study was to compare the efficacy between CDT and anticoagulation alone.

\section{Secondary End Point}

1. Technical success of CDT, defined as complete lysis of the thrombus of the vein, without distal thromboembolic complications

2. Thrombolysis-induced hemorrhagic complications

3. Duration of hospital admission.

\section{Study Design}

It was a prospective and comparative study.

\section{Patients}

All patients presented with lower limb DVT who met the inclusion criteria in the year 2012 were included in this study.

\section{Eligibility Criteria}

\section{Inclusion Criteria}

1. Men and women between 12 and 85 years of age

2. Patients with recent (between 1 and 8 weeks) lower limb DVT

3. Patients understand the nature of the procedure and provide written informed consent before enrollment in the study.

\section{Exclusion Criteria}

1. Patients with DVT of more than 8 weeks

2. Patients for whom antiplatelet therapy, anticoagulants, or thrombolytic drugs are contraindicated

3. Recent ( $<6$ weeks) ischemic stroke or cerebral bleeding

4. Patients with recent ( $<6$ weeks) major surgery

5. Severe hypertension (diastolic blood pressure greater than $110 \mathrm{~mm} \mathrm{Hg}$, systolic blood pressure greater than $200 \mathrm{~mm} \mathrm{Hg}$ )

6. Patients with a history of prior life-threatening reaction to contrast medium

7. Patients with uncorrected bleeding disorders (gastrointestinal ulcer, menorrhagia, liver failure)
8. Women with child-bearing potential not taking adequate contraceptives or currently breastfeeding

9. Patients considered hemodynamically unstable at the onset of the procedure

10. Patients who refuse treatment

11. Patients younger than 12 years or older than 85 years

12. Severe comorbid condition with a life expectancy of less than 1 month

13. Hemoglobin $<9 \mathrm{mg} / \mathrm{dL}$, international normalized ratio (INR) $>1.6$ before warfarin is initiated, and platelet count $<100,000 / \mathrm{mL}$.

\section{Ethics}

The study protocol was approved by the Ethics Committee of the respective authority of our institution. Written informed consent will be obtained from all patients, before inclusion in the study.

\section{Adverse and Serious Adverse Events}

Adverse events (AEs) are defined as any undesirable experience occurring to a participant during the study, whether or not considered related to the investigational device. This definition includes events occurring during hospital stay right up to 30 days of follow-up.

Underlying disease that was present at the time of enrollment is not reported as an $\mathrm{AE}$, but any increase in the severity of the underlying disease will be reported as an $\mathrm{AE}$.

All AEs will be monitored from the time of enrollment through the 30-day follow-up visit.

Clinical events to be considered and reported as serious AEs include:

- Death

- Myocardial infarction

- Stroke

- Bleeding complication requiring interruption or ending of thrombolysis

- Bleeding complication requiring surgical intervention

- Bleeding complication requiring transfusion.

- Clinical events to be considered and reported as minimal AEs include:

- Groin hematoma

- Bleeding complication not requiring interruption or ending of thrombolysis, surgical intervention.

Written pro forma was filled up during inclusion of patients which contained epidemiological information (age, sex, occupation, and place), questionnaires for risk factor evaluation (smoking, drug history, malignancy, comorbid condition, hypercoagulable state), information of clinical examinations (limb involvement).

\section{Investigations}

All patients will be investigated with the following tests:

1. Complete hemogram

2. Bleeding time, clotting time (CT), prothrombin time, and activated plasma thromboplastin time 
3. Renal function test, liver function test

4. Work-up for hypercoagulation state (if affordable)

5. Duplex venography/ultrasound abdomen.

\section{Intervention}

After admission with proximal lower limb DVT, patients were treated according to the unit protocol as there was no fixed institutional protocol regarding the treatment of proximal DVT. Investigators collected data prospectively, compiled, and finally compared between the patients who received CDT with patients who received anticoagulation alone. As all patients with proximal lower limb DVT who met the inclusion and exclusion criteria in the investigating unit were treated with CDT and these data were compared with the other unit patients not being treated with CDT.

\section{Treatment Arm A (Catheter-Directed Thrombolysis)}

In our study, popliteal vein cannulation under ultrasound guidance was done to access the lesion on the same site of the DVT. Peripheral angiogram was done in this group through popliteal vein approach. Before the CDT procedure, low molecular weight heparin (LMWH) was discontinued for at least 8 hours, and oral anticoagulants were discontinued to obtain an INR $<1.5$. At the start of CDT, an intravenous bolus dose of unfractionated heparin (UFH), 5,000 U, followed by a continuous intravenous UFH infusion at the rate of $1,000 \mathrm{U} / \mathrm{h}$ was given. ${ }^{14}$ At first, thrombus was manually aspirated by using Judkin's right guiding catheter (JR 3.0 6F, Cordis, Miami, FL) and then CDT was done by inserting multipurpose catheter in situ with injection of Streptokinase (STK) was given for prolonged period till satisfactory results came. Two-thirds of the total doses of STK ( 1 lakh units/h) were given through catheter and other one-third of STK was given through intravenous sheath. UFH was infused through the intravenous sheath. Check angiogram was done at regular interval. The difference between the pre- and postlysis thrombus scores divided by the prelysis score gave the grade of thrombolysis; grade $\mathrm{I}=<50 \%$; grade II $=50-90 \%$, and grade III = complete thrombolysis. ${ }^{15}$

\section{Treatment Arm B (Anticoagulation Alone)}

Patients were treated with UFH infusion of $1,000 \mathrm{IU} / \mathrm{h}$ for first 48 hours. All these cases also received bolus of UFH (5,000 IU 6 hourly) for another 5 days or LMWH $1 \mathrm{mg} / \mathrm{kg}$ body weight for another 5 days. ${ }^{1}$

\section{Oral Anticoagulant}

Patients of both treatment arms received oral warfarin or Nicoumalone for 6 months, started since day 1 of the treatment in treatment arm B or after CDT in treatment arm A. The INR was maintained within 2 to 3 days. ${ }^{1}$

\section{Follow-Up}

Patients of both the treatment arms followed-up during their hospital stay, monthly till 6 months. After 6 months, deep venous patency was investigated by using duplex ultrasound. The PTS was assessed using the Villalta scale, which consists of five patient-rated venous symptoms (pain, cramps, heaviness, paresthesia, and pruritus) and six clinician-rated physical signs (pretibial edema, skin induration, hyperpigmentation, pain during calf compression, venous ectasia, and redness). Each is rated on a four-point scale (0-none, 1-mild, 2-moderate, and 3 -severe). Points are totaled to produce an overall score (range, 0-33). Subjects were classified as having PTS if their score was $>5$ or if a venous ulcer developed in the leg with DVT. ${ }^{16}$ The Villalta scale is a reliable, validated, and responsive measure of PTS. ${ }^{17}$

\section{Statistical Analysis}

As it is a comparative study, paired value will be analyzed with paired " $T$ " test, chi-square test, or Student $t$-test by using SPSS software (Chicago, IL). The $p$ value of $<0.05$ will be considered as statistically significant.

\section{Results}

Total 64 patients were admitted with DVT within the study period and among them 4 patients had upper limb DVT and were excluded from the study. Among 60 patients, equal numbers of patients were treated in both treatment arms after considering the exclusion criteria. In the treatment arm $\mathrm{A}$ (CDT group), two patients died during treatment and another patient did not complete therapy, so they were excluded from study. In the treatment arm B (routine anticoagulation group), two patients died during treatment and were excluded from the study. Among the patients included in this study, two from each group were lost during follow-up, so finally 25 and 26 patients were included in the treatment arms $A$ and $B$, respectively, data were collected, compiled, and analyzed.

\section{Baseline Demographic Profile}

The mean age of treatment arm A was significantly ( $p<0.03$ ) less than that of treatment arm B (39 $[ \pm 16]$ years vs. 53 $[ \pm 17]$ years). Both the groups had more number of male patients without any statistical significance. Mean duration of

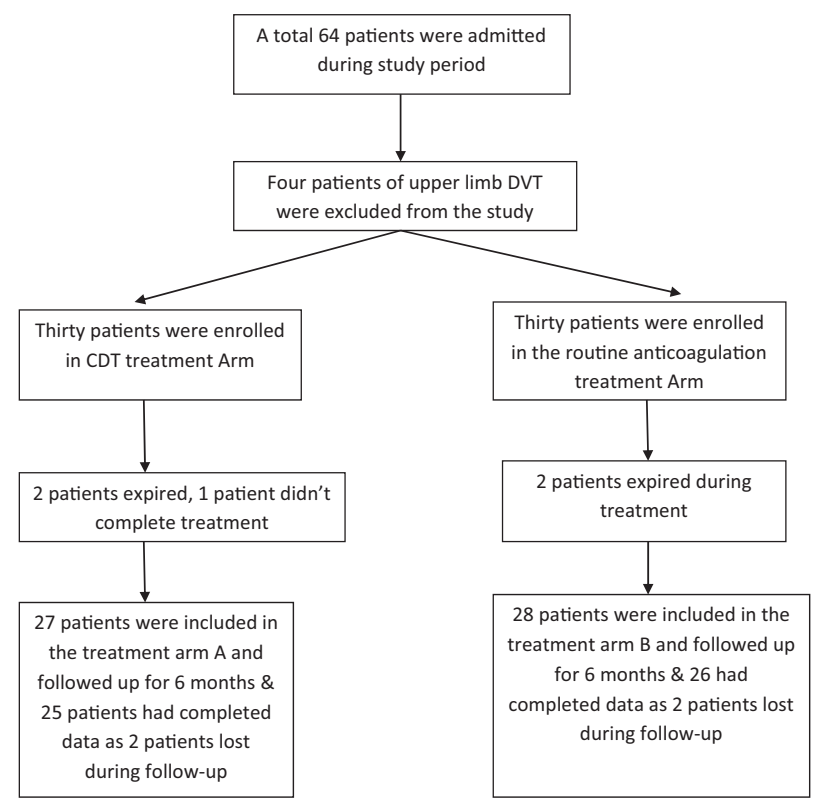

Fig. 1 Flow chart of the study. 
Table 1 Baseline demographic and clinical profile of study groups

\begin{tabular}{|c|c|c|c|}
\hline Variable & $\begin{array}{l}\text { Treatment arm A } \\
(n=27)\end{array}$ & $\begin{array}{l}\text { Treatment arm B } \\
(n=28)\end{array}$ & $p$ Value \\
\hline Age (mean, range) (y) & $39( \pm 16), 20-62$ & $53( \pm 17), 27-80$ & $<0.03$ \\
\hline $\operatorname{Sex}(M: F)$ & $14: 13$ & $16: 12$ & NS \\
\hline \multicolumn{4}{|l|}{ Risk factors } \\
\hline $\mathrm{MI} / \mathrm{LVD}$ & $4(15 \%)$ & $5(18 \%)$ & NS \\
\hline Smoking & $3(11 \%)$ & $4(14 \%)$ & NS \\
\hline Diabetes & $5(18 \%)$ & $3(11 \%)$ & NS \\
\hline Malignancy & $2(7 \%)$ & $6(21 \%)$ & NS \\
\hline Trauma/fracture/prolonged bed ridden & $6(22 \%)$ & $4(14 \%)$ & NS \\
\hline Pregnancy/postpartum & $5(15 \%)$ & $3(11 \%)$ & NS \\
\hline Others (alcoholic/seizure disorder, polio, nephritic syndrome) & $6(22 \%)$ & $10(36 \%)$ & NS \\
\hline Idiopathic & $2(7 \%)$ & $6(21 \%)$ & NS \\
\hline Duration of symptoms (mean, range) (d) & $12 \pm 8,2-30$ & $21 \pm 20,3-56$ & $<0.01$ \\
\hline \multicolumn{4}{|l|}{ Affected limb } \\
\hline Left & $5(18 \%)$ & $8(28 \%)$ & NS \\
\hline Right & $18(64 \%)$ & $16(57 \%)$ & NS \\
\hline Bilateral & $5(18 \%)$ & $4(14 \%)$ & NS \\
\hline \multicolumn{4}{|l|}{ Thrombosis location } \\
\hline Iliofemoral & $24(89 \%)$ & $19(68 \%)$ & NS \\
\hline Femoropopliteal & $18(67 \%)$ & $18(64 \%)$ & NS \\
\hline IVC/renal vein involvement & 7 (RVT-3) (26\%) & $3(7 \%)$ & 0.04 \\
\hline Duration of hospital stay (mean, range) (d) & $5 \pm 1.3,3-7$ & $4.8 \pm 1.4,3-8$ & NS \\
\hline Pulmonary embolism during treatment & $4(15 \%)$ & $6(21 \%)$ & NS \\
\hline Death during treatment & $2(7 \%)$ & $2(7 \%)$ & NS \\
\hline
\end{tabular}

Abbreviations: IVC, inferior vena cava; MI/LVD, myocardial infarction/left ventricular dysfunction; NS, nonsignificant; RVT, renal vein thrombosis in 3 patients.

symptoms at presentation was also significantly $(p<0.01)$ less in the treatment arm A ( $12 \pm 8$ days) than in the treatment arm $B(21 \pm 20$ days) ( - Table $\mathbf{1})$.

\section{Risk Factors of DVT in Our Study Groups}

The most common risk factor of our patients was prolonged bed ridden state and mostly due to trauma or fracture of lower limb (18\%) which was followed by ischemic heart disease/left ventricular dysfunction (16\%), diabetes (15\%), postpartum state $(15 \%)$, systemic malignancy $(15 \%)$, and smoking (13\%). Twenty-nine percent of patients had other risk factors such as nephritic syndrome, seizure disorder, dyslipidemia, alcoholism, poliomyelitis, etc. Fifteen percent of our patients had no definite risk factors for DVT. But there were no statistically significant differences seen between the study and control groups (-Table $\mathbf{1}$ ).

\section{Distribution and Location of DVT}

In our study patients, $24 \%$ patients had involvement of left lower limb and $62 \%$ had involvement of right lower limb, whereas $16 \%$ of our patients had involvement of both lower limbs. Seventy-eight percent of our patients had proximal iliofemoral DVT. Sixty-five percent of patients had femoro- popliteal DVT. So, there were few patients who had extension of DVT from proximal common iliac vein to distally tibiopopliteal vein. Eighteen percent of our patients had extension of DVT up to inferior vena cava (IVC) and/or renal vein (three patients). Distribution of DVT among the study groups was comparable with no statistically significant differences as shown in - Table 1.

\section{Duration of Stay in Intensive Care Unit}

There was statistically insignificant prolonged stay seen in CDT group ( $5 \pm 1.3$ days) than in the routine anticoagulation $\operatorname{arm}(4.8 \pm 1.4$ days $)$. There were no statistically significant differences in mortality rate and development of pulmonary embolism during therapy in both the groups (-Table 1).

\section{Treatment Outcome after Catheter-Directed Thrombolysis}

Mean duration of CDT with STK infusion was approximately $108( \pm 32)$ hours and so the average dose of STK given to a patient is approximately 10 to 11 million units. All of our patients received CDT along with mechanical thrombolysis which was done just before thrombolysis. Thirty-seven 
Table 2 Treatment outcome after CDT

\begin{tabular}{|c|c|}
\hline Variable & Value \\
\hline Duration of CDT (mean, range) (h) & $\begin{array}{l}108 \pm 32 \\
42-144\end{array}$ \\
\hline Dose of streptokinase (mean, range) (MU) & $11,4-14$ \\
\hline \multicolumn{2}{|l|}{$\begin{array}{l}\text { Immediate lysis grade after CDT }+ \text { mechanical } \\
\text { thrombectomy }(n=27)\end{array}$} \\
\hline Grade I & $0(0 \%)$ \\
\hline Grade II & $17(63 \%)$ \\
\hline Grade III & $10(37 \%)$ \\
\hline \multicolumn{2}{|l|}{ Further intervention } \\
\hline Only CDT & $10(37 \%)$ \\
\hline CDT + PTA & $11(41 \%)$ \\
\hline CDT + PTA and stenting & $6(22 \%)$ \\
\hline IVC filter implantation & $5(18 \%)$ \\
\hline \multicolumn{2}{|l|}{ Adverse events } \\
\hline \multicolumn{2}{|l|}{ SAE } \\
\hline Pulmonary embolism & $4(15 \%)$ \\
\hline Anemia requiring blood transfusion & $4(15 \%)$ \\
\hline \multicolumn{2}{|l|}{ MAE } \\
\hline Local site bleeding/hematoma & $13(48 \%)$ \\
\hline Anemia not requiring blood transfusion & $4(15 \%)$ \\
\hline Thrombocytopenia & $6(22 \%)$ \\
\hline
\end{tabular}

Abbreviations: CDT, catheter-directed thrombolysis; IVC, inferior vena cava; PTA, percutaneous transluminal angioplasty; SAE, serious adverse events; $\mathrm{MAE}=$ minimal adverse events

percent of CDT group patients had grade III lysis at the end of the CDT, whereas grade II lysis in 63\%. Percutaneous transluminal angioplasty (PTA) along with CDT and mechanical thrombectomy was done in patients with partial clot lysis following CDT. PTA and stenting was done in $22 \%$ and IVC filter implantation was done in $18 \%$ of CDT group patients. Among seven patients, who had thrombus extension into IVC in CDT group, five patients had IVC filter implantation. IVC filter was implanted in patients who had developed pulmonary embolism and who had large thrombus burden or had progressive extension of thrombus into IVC. Among the five cases with retrievable IVC filter implantation, in four cases the filter was successfully retrieved except one case where it could not be retrieved. Three of our patients had MayThurner syndrome and was having PTA and stenting of left common iliac vein (-Table 2 ).

\section{Adverse Events of Catheter-Directed Thrombolysis}

Serious AEs as per definition in our study were seen in $24 \%$ of CDT group patients and among these, $8 \%$ patients had diagnosed pulmonary embolism during CDT by CT pulmonary angiogram. These patients had thrombus extension into IVC and IVC filter was implanted. Among the four patients who suffered from acute pulmonary embolism, two patients died. In another two patients, IVC filter was inserted. These patients were elderly female and one of them was treated previously for breast carcinoma and both of them did not have thrombus extension into IVC. About $15 \%$ of patients developed severe anemia after CDT with mechanical thrombosuction and received blood transfusion as they had significant drop of hemoglobin following repeated mechanical thromboaspiration with hemoglobin level less than $8 \mathrm{~g} / \mathrm{dL}$. Among minor AEs, $48 \%$ patients had local site bleeding or hematoma and $15 \%$ had anemia not requiring blood transfusion. About $22 \%$ of patients had thrombocytopenia without requirement for platelet transfusion started after 4 days of treatment (-Table 2).

\section{Efficacy Outcome at 6 Months after Follow-Up}

After 6 months of follow-up, 80\% of patients in CDT group had patent lower limb vein diagnosed through duplex venography and $23 \%$ of patients in anticoagulation alone group had patent leg vein. PTS diagnosed by Villalta scoring system was seen in $20 \%$ of patents in treatment arm $A$ and $77 \%$ of patients in treatment arm B. Both these results were found to be statistically significant (-Table $\mathbf{3}$ ).

\section{Discussion}

The management of patients with acute DVT with early thrombus removal by CDT and/or mechanical thromboaspiration currently becoming standard of care in the management of proximal DVT as it showed promising results, rapid removal of clot burden, and decreased incidence of PTS though currently considered as $2 \mathrm{~B}$ recommendation. ${ }^{1}$ Several studies have reported that 25 to $82 \%$ of patients with iliofemoral DVT who were treated with anticoagulant alone went on to suffer from the PTS. ${ }^{8}$ Though there are several studies which used either urokinase or alteplase as thrombolytic agents for the management of CDT, ${ }^{7-10}$ but it is the second study where STK is being used for this purpose. ${ }^{11}$ Systemic use of thrombolytic agents has been shown to be no more effective than heparin in achieving clot lysis in small dose as high-dose systemic thrombolysis results in a significantly better short- and long-term clinical outcomes than conventional heparin/anticoagulation therapy but at the expense of a serious increase in major bleeding and pulmonary emboli. ${ }^{1,2}$ The use of local and regional thrombolysis (infusing the thrombolytic agent in a dorsal foot vein or popliteal vein)

Table 3 Efficacy outcome at 6 months follow-up

\begin{tabular}{|l|l|l|l|}
\hline Variable & $\begin{array}{l}\text { Treatment } \\
\text { arm A } \\
(\boldsymbol{n}=25)\end{array}$ & $\begin{array}{l}\text { Treatment } \\
\text { arm B } \\
(\boldsymbol{n}=26)\end{array}$ & $p$ Value \\
\hline $\begin{array}{l}\text { Venous patency } \\
\begin{array}{l}>90 \% \text { clearance) at } \\
6 \text { mo (by duplex } \\
\text { venography) }\end{array}\end{array}$ & $20(80 \%)$ & $7(23 \%)$ & $<0.01$ \\
\hline $\begin{array}{l}\text { PTS (Villalta } \\
\text { score }>5)\end{array}$ & $5(20 \%)$ & $19(77 \%)$ & $<0.01$ \\
\hline
\end{tabular}

Abbreviation: PTS, postthrombotic syndrome. 
demonstrated significantly fewer clinical symptoms of PTS with fewer complications due to thrombolytic agents. ${ }^{11} \mathrm{CDT}$ therapy has been studied recently and has been shown to be safe and effective in achieving early, intermediate, and longterm venous patencies. ${ }^{18-22}$ The ipsilateral popliteal venous approach is preferred because it is often difficult to penetrate an occluded superior iliofemoral vein from the internal jugular vein or the contralateral common femoral vein, and venous valves may prevent safe catheterization. ${ }^{11}$ This approach also clears the distal veins. Numerous studies have reported positive outcomes for CDT in acute DVT. ${ }^{12-22}$ Mewissen et $\mathrm{al}^{15}$ documented a complete resolution rate of $31 \%$, a partial rate of $52 \%$, and a failure rate of $17 \%$, whereas other studies have found 6 to 12 months patency in 38 to $50 \%$ after standard treatment. These efficacy estimates are consistent with our study findings. In our study, though $37 \%$ of patients had grade III lysis after CDT and mechanical thromboaspiration but 6 months' patency rate was $80 \%$ after CDT, PTA, and/or stenting. Though our study has similarity with the previous study of Elsharawy and Elzayat ${ }^{11}$ as in both studies STK was used, local popliteal vein was taken, but mean duration of STK infusion was longer which was similar to CaVenT study where alteplase was used. ${ }^{12}$ As our study allowed patients with up to 8 weeks history of DVT symptoms compared with $<10$ days in the earlier study, ${ }^{11}$ this may be the cause for partial clot lysis. In our study, only $20 \%$ of CDT group developed PTS, whereas $77 \%$ of patients in anticoagulation alone group developed PTS which was comparable from previous study. Though numerous devices have been recently developed for pharmacomechanical thrombolysis, but we performed a mechanical thrombectomy by manual aspiration due to financial constrain in our patients. However, a study by Lin et al showed no statistical difference between thrombus removal rates for the pharmacomechanical thrombectomy using the AngioJet system and CDT. ${ }^{21}$ In our study cohort, we used a retrievable IVC filter (Optease, Cordis) in five $(18 \%)$ of the patients to prevent thrombus embolization during the procedures. Currently, prophylactic IVC filter insertion is recommended in patients with a free-floating IVC thrombus that is longer than $5 \mathrm{~cm}^{22}$ The reported complications of CDT are bleeding, pulmonary embolism, and death. ${ }^{20}$ The incidence of pulmonary embolism after CDT in our study was $15 \%$, which was similar to the 10 to $26 \%$ previously reported. ${ }^{22-24}$ In the present study, there was two deaths, $15 \%$ patients had severe anemia due to aspiration of blood and thrombus during mechanical thromboaspiration and required blood transfusion. About $48 \%$ of patients developed a minor bleeding complication such as oozing from local site or hematoma which was more than the previous studies $^{2,11}$ and no case had severe life-threatening bleeding. In contrast to previous studies, ${ }^{11}$ our study had $24 \%$ of patients with thrombocytopenia developed after 4 to 5 days of CDT. This may be due to heparin-induced thrombocytopenia.

\section{Limitations}

First, it was a comparative trial so chance of selection bias was more. Second, sample size in our study was small.

\section{Conclusion}

This report represents the second clinical study of CDT using STK and heparin infusion versus heparin/LMWH alone for treatment of DVT. We conclude that CDT and conventional manual aspiration thrombectomy is an effective treatment for lower extremity DVT, and the clinical results achieved were satisfactory. An additional CDT increased patency at 6 months after iliofemoral DVT, from 23 to $80 \%$. Though, STK infusion can be used safely for CDT up to 6 days to achieve good results as it increases only minor bleeding rate, but addition of UFH infusion can cause thrombocytopenia. So, daily monitoring of complete blood counts is needed during CDT. There was a serious concern as two deaths had occurred due to suspected pulmonary embolism following CDT in our study, so whether prophylactic IVC filter implantation is mandatory before giving CDT and mechanical thromboaspiration needs to be confirmed in further large randomized control trial.

\section{Contribution of Authors}

Soumya Patra and C.M. Nagesh planned the study. Soumya Patra designed the protocol, collected and analyzed the data, reviewed the literatures, and drafted the article. Babu Reddy, B.C. Srinivas and C.N. Manjunath were involved in the management of the patients. All authors approved the final version of the article.

\section{Conflict of Interest}

None.

\section{References}

1 Jaff MR, McMurtry MS, Archer SL, et al; American Heart Association Council on Cardiopulmonary, Critical Care, Perioperative and Resuscitation. ; American Heart Association Council on Peripheral Vascular Disease. ; American Heart Association Council on Arteriosclerosis, Thrombosis and Vascular Biology. Management of massive and submassive pulmonary embolism, iliofemoral deep vein thrombosis, and chronic thromboembolic pulmonary hypertension: a scientific statement from the American Heart Association. Circulation 2011;123(16):1788-1830

2 Cohen AT, Agnelli G, Anderson FA, et al; VTE Impact Assessment Group in Europe (VITAE). Venous thromboembolism (VTE) in Europe. The number of VTE events and associated morbidity and mortality. Thromb Haemost 2007;98(4):756-764

3 Hirsh J, Hoak J. Management of deep vein thrombosis and pulmonary embolism. A statement for healthcare professionals. Council on Thrombosis (in consultation with the Council on Cardiovascular Radiology), American Heart Association. Circulation 1996;93(12): 2212-2245

4 Gauci BN, Powell JT, Hunt BJ, et al. The feasibility of catheterdirected thrombolysis for acute deep vein thrombosis: a regional perspective. Phlebology 2011;26(3):94-101

5 Lin PH, Ochoa LN, Duffy P. Catheter-directed thrombectomy and thrombolysis for symptomatic lower-extremity deep vein thrombosis: review of current interventional treatment strategies. Perspect Vasc Surg Endovasc Ther 2010;22(3):152-163

6 Prandoni P, Lensing AW, Prins MH, et al. Below-knee elastic compression stockings to prevent the post-thrombotic syndrome: 
a randomized, controlled trial. Ann Intern Med 2004;141(4): 249-256

7 Comerota AJ. The ATTRACT trial: rationale for early intervention for iliofemoral DVT. Perspect Vasc Surg Endovasc Ther 2009;21(4): 221-224, quiz 224-225

8 Comerota AJ, Paolini D. Treatment of acute iliofemoral deep venous thrombosis: a strategy of thrombus removal. Eur J Vasc Endovasc Surg 2007;33(3):351-360, discussion 361-362

9 Patterson BO, Hinchliffe R, Loftus IM, Thompson MM, Holt PJ. Indications for catheter-directed thrombolysis in the management of acute proximal deep venous thrombosis. Arterioscler Thromb Vasc Biol 2010;30(4):669-674

10 Enden T, Kløw NE, Sandvik L, et al. Catheter-directed thrombolysis vs. anticoagulant therapy alone in deep vein thrombosis: results of an open randomized, controlled trial reporting on short-term patency. J Thromb Haemost 2009;7(8):1268-1275

11 Elsharawy M, Elzayat E. Early results of thrombolysis vs anticoagulation in iliofemoral venous thrombosis. A randomised clinical trial. Eur J Vasc Endovasc Surg 2002;24(3): 209-214

12 Enden T, Haig Y, Kløw NE, et al; CaVenT Study Group. Long-term outcome after additional catheter-directed thrombolysis versus standard treatment for acute iliofemoral deep vein thrombosis (the CaVenT study): a randomised controlled trial. Lancet 2012; 379(9810):31-38

13 Enden T, Haig Y, Kløw NE, et al. CaVenT Study Group. Long-term outcome after additional catheter-directed thrombolysis versus standard treatment for acute iliofemoral deep vein thrombosis (the CaVenT study): a randomised controlled trial. Lancet 2012; 379(9810):31-38

14 Kearon C, Kahn SR, Agnelli G, Goldhaber S, Raskob GE, Comerota AJ; American College of Chest Physicians. Antithrombotic therapy for venous thromboembolic disease: American College of Chest Physicians Evidence-Based Clinical Practice Guidelines (8th Edition). Chest 2008;133(6, Suppl):454S-545S
15 Mewissen MW, Seabrook GR, Meissner MH, Cynamon J, Labropoulos N, Haughton SH. Catheter-directed thrombolysis for lower extremity deep venous thrombosis: report of a national multicenter registry. Radiology 1999;211(1):39-49

16 Kahn SR. Measurement properties of the Villalta scale to define and classify the severity of the post-thrombotic syndrome. J Thromb Haemost 2009;7(5):884-888

17 Broholm R, Sillesen H, Damsgaard MT, et al. Postthrombotic syndrome and quality of life in patients with iliofemoral venous thrombosis treated with catheter-directed thrombolysis. J Vasc Surg 2011;54(6, Suppl):18S-25S

18 Grewal NK, Martinez JT, Andrews L, Comerota AJ. Quantity of clot lysed after catheter-directed thrombolysis for iliofemoral deep venous thrombosis correlates with postthrombotic morbidity. J Vasc Surg 2010;51(5):1209-1214

19 Pianta MJ, Thomson KR. Catheter-directed thrombolysis of lower limb thrombosis. Cardiovasc Intervent Radiol 2011;34(1):25-36

20 Meissner MH, Wakefield TW, Ascher E, et al. Acute venous disease: venous thrombosis and venous trauma. J Vasc Surg 2007;46(Suppl S ):25S-53S

21 Lin PH, Zhou W, Dardik A, et al. Catheter-direct thrombolysis versus pharmacomechanical thrombectomy for treatment of symptomatic lower extremity deep venous thrombosis. Am J Surg 2006;192(6):782-788

22 Patel NH, Stookey KR, Ketcham DB, Cragg AH. Endovascular management of acute extensive iliofemoral deep venous thrombosis caused by May-Thurner syndrome. J Vasc Interv Radiol 2000; 11(10):1297-1302

23 Goldhaber SZ, Hirsch DR, MacDougall RC, Polak JF, Creager MA. Bolus recombinant urokinase versus heparin in deep venous thrombosis: a randomized controlled trial. Am Heart J 1996; 132(2 Pt 1):314-318

24 Schweizer J, Kirch W, Koch R, et al. Short- and long-term results after thrombolytic treatment of deep venous thrombosis. J Am Coll Cardiol 2000;36(4):1336-1343 